\title{
Practical Approaches for High-Risk Surgical Patients with Acute Cholecystitis: The Percutaneous Approach versus Endoscopic Alternatives
}

\author{
Rungsun Rerknimitr ${ }^{1,2}$ and Khanh Cong Pham ${ }^{3}$ \\ ${ }^{1}$ Division of Gastroenterology, Department of Medicine, Faculty of Medicine, Chulalongkorn University and King Chulalongkorn Memorial \\ Hospital, Bangkok, Thailand, ${ }^{2}$ Center of Excellence for Innovation and Endoscopy in Gastrointestinal Oncology, Chulalongkorn University, \\ Bangkok, Thailand, ${ }^{3}$ Department of Endoscopy, University Medical Center, Ho Chi Minh, Vietnam
}

In high-risk surgical patients with acute cholecystitis who are not candidates for early laparoscopic cholecystectomy, gallbladder (GB) drainage is an alternative treatment option. Percutaneous transhepatic gallbladder drainage (PTGBD) is a recommended first line intervention because of its high efficacy and feasibility in most centers. However, with the advent of endoscopic accessories and technology, endoscopic GB drainage has been chosen as a more favorable choice by endoscopists. Endoscopic transpapillary gallbladder drainage (ETGBD) can be performed under either fluoroscopic or peroral cholangioscopic guidance via endoscopic retrograde cholangiopancreatography by the transpapillary placement of a long double-pigtail stent. In a patient with common bile duct stones, this procedure is accompanied with stone removal. ETGBD is especially useful for acute cholecystitis patients who are contraindicated for PTGBD or those with severe coagulopathy, thrombocytopenia, and abnormal anatomy. Moreover, the advantage of ETGBD is its preservation of the external GB structure. Thereby it would not disturb the future cholecystectomy. Recently, endoscopic ultrasoundguided gallbladder drainage (EUS-GBD) using plastic, fully covered metallic, or lumen-apposing metal stents transmurally has emerged as a modality for GB drainage with higher technical and clinical success rates. EUS-GBD can provide a more permanent GB drainage than PTGBD and ETGBD. Clin Endosc 2020;53:678-685

Key Words: Acute cholecystitis; Endoscopic retrograde cholangiopancreatography; Endoscopic ultrasound; Gallbladder drainage

\section{INTRODUCTION}

Acute cholecystitis (AC) is well perceived as acute inflammation of the gallbladder (GB) and usually develops after cystic duct obstruction caused by stones, or blockage from an object such as a biliary metallic stent or tumor. AC can cause some severe complications such as empyema, gangrenous

Received: September 15, 2019 Revised: October 21, 2019

Accepted: October 25, 2019

Correspondence: Rungsun Rerknimitr

Division of Gastroenterology, Department of Medicine, Faculty of Medicine, Chulalongkorn University and King Chulalongkorn Memorial Hospital, 1873 Rama IV Rd, Pathum Wan, Pathum Wan District, Bangkok 10330, Thailand Tel: +66-2-256-4265, Fax: +66-2-252-7839, E-mail: ERCP@live.com ORCID: https://orcid.org/0000-0001-6866-6886

(c) This is an Open Access article distributed under the terms of the Creative Commons Attribution Non-Commercial License (http://creativecommons.org/ licenses/by-nc/3.0) which permits unrestricted non-commercial use, distribution, and reproduction in any medium, provided the original work is properly cited. cholecystitis, perforated GB, and septic shock. AC can also be life-threatening, particularly in elderly patients, because of concurrent illnesses and its atypical clinical presentations. Recurrent attacks of AC can lead to a condition called chronic cholecystitis, which has been well characterized by the presence of GB wall thickening, atrophic lumen, and adhesion between the GB with surrounding structures.

Laparoscopic cholecystectomy (LC) is considered the gold standard for $\mathrm{AC}$ and has superseded open surgery for over a few decades, as it yields similar efficacy, but a lower rate of morbidities with the expected results of quicker recovery, shortened hospitalization, and improved quality of life. ${ }^{1-3}$ The timing of LC has been a subject of debate for several years. A meta-analysis ${ }^{4}$ from 77 case-control series including 26,549 patients showed statistically significant lower incidences in mortality rate and surgery-related adverse events including bile leakage, biliary tract injuries, and infection of the incision 
site in those who underwent early LC than delayed LC. ${ }^{4}$ In addition, the length of hospitalization and blood loss were lower with early LC. Severe bile duct-related complications in the early and delayed group were $0.26 \%$ and $0.53 \%$, respectively, (odds ratio $[\mathrm{OR}], 0.49 ; p<0.001$ ). The results from this meta-analysis showed that early LC is clearly superior to delayed LC and should be the standard of care in the management (in the setting) of $\mathrm{AC}^{4}$

Unfortunately, many moderate and severe AC patients, especially those with high co-morbid illnesses (Charlson Comorbidity Index $\geq 6$ and American Society of Anesthesiologists Physical Status $\geq 3$ ), are not suitable for early LC. Therefore, GB drainage is necessary before considering elective surgery. This is to prevent GB gangrene and/or perforation and reduce the risk of bacteremia. Traditionally, percutaneous transhepatic gallbladder drainage (PTGBD) is recommended as the primary strategy to decompress the GB. Recently, alternative endoscopic GB drainage has gained popularity as many patients are under the care of endoscopists. Endoscopic GB drainage includes a transpapillary and transmural approach. Transmural GB drainage requires an endoscopic ultrasound (EUS) with linear echoendoscope and is called EUS-guided gallbladder drainage (EUS-GBD)., ${ }^{5,6}$

In this review, we describe the current evidence for $\mathrm{AC}$ management and the roles of endoscopic GB drainage for AC (both endoscopic retrograde cholangiopancreatography [ERCP] and EUS guidance) compared with PTGBD. Finally, we propose the future directions for different approaches based on the severity of AC, surgical expertise for LC, and patient candidacy for surgery.

\section{DIAGNOSIS AND CLASSIFICATION OF ACUTE CHOLECYSTITIS}

The recently published Tokyo Guideline 2018 has proposed the diagnostic criteria for AC in three components: (1) signs of localized peritonitis including Murphy's sign; (2) evidence of systemic inflammation including high temperatures, higher than normal C-reactive protein levels and leukocytosis; and (3) compatible abdominal images including ultrasound, computed tomography scan, or magnetic resonance imaging. The presence of criteria A and B constitutes a suspected diagnosis, whereas the presence of all three criteria confirms a definitive diagnosis. ${ }^{7}$

For AC severity grading, Grade I AC is defined as mild inflammation of the GB in healthy patients with normal function of other organs. Grade II AC is diagnosed as a patient with significant leukocytosis, tenderness of the $\mathrm{GB}$, more than 3 days of AC-related symptoms, or those with local inflamma- tion. Grade III is diagnosed as a AC-associated organ failure and/or tissue hypoperfusion. ${ }^{7}$

\section{STRATEGICAL MANAGEMENT BY THE SEVERITY OF GALLBLADDER INFLAMMATION}

In accordance with the instructions of the Tokyo guideline 2018, the treatment plan is dependent on AC severity. Early LC is recommended in grade I, II, and III AC in patients who are surgical candidates. However, LC should be performed by a skillful surgeon in AC patients with grade II and III. If the patient is not stable enough for surgery, conservative management with intravenous hydration and antibiotics, pain management, etc., should be initiated and surgery taking place at a later time. Early GB drainage should be performed in patients with grade II AC in whom conservative management failed or those with high surgical risks with grade III (Fig. 1). ${ }^{6}$ GB drainage can be done by either percutaneous cholecystostomy (percutaneous transhepatic GB drainage) or endoscopic drainage. Endoscopic transpapillary gallbladder drainage (ETGBD) in which the GB can be drained by a naso-biliary tube (5-7 F) or double-pigtail stent (7-10 F) through ERCP. Recently, EUSGBD was deemed a promising technique for GB drainage in patients with $\mathrm{AC}^{3}$

\section{GALLBLADDER DRAINAGE}

\section{Percutaneous transhepatic gallbladder drainage}

PTGBD is traditionally the first-line approach in high-risk surgical AC patients because PTGBD is a minimally invasive technique with a low complication. ${ }^{3}$ PTGBD has a high clinical success rate for the temporary decompression of the GB (86\%-97\%). ${ }^{8.9}$ Applying PTGBD before scheduled LC for moderate and severe AC not only decreases the operating time, intraoperative bleeding, and length of postoperative hospitalization but also decreases the conversion rate to laparotomy and postoperative complications. It is recommended as the preferred therapy for elderly patients especially those with moderate to severe AC. Lee et al. reported a study in 85 moderate to severe AC patients whom were arranged in 2 groups: the 1st group included PTGBD with scheduled LC, and the 2 nd group received early LC without GB drainage. ${ }^{10}$ The operative time was significantly decreased in the 1st group (73.4 $\mathrm{min}$ in the 1st group vs. $91.4 \mathrm{~min}$ in the 2 nd group, $p=0.012){ }^{10}$ A meta-analysis covering 9 randomized controlled trials included 1,000 moderate to severe AC patients with high surgical risks (>60 years old). It concluded that LC scheduled after 
PTGBD had significant better effects on the operative duration, estimation of intraoperative bleeding, conversion rate to laparotomy, postoperative complication morbidity, and postoperative hospitalization than direct LC. Tan et al. conducted a prospective study which compared treatment between early and scheduled LC performed 6 weeks after PTGBD. ${ }^{11}$ One hundred and fifty AC patients with grade II were divided into two groups (early LC or PTGBD + scheduled LC). The conversion rate of the early LC group was remarkably higher than the PTGBD + scheduled LC group ( $24 \%$ vs. $2.7 \%, p<0.001){ }^{11}$ Moreover, the early LC group had a longer mean operative time (88 $\mathrm{min}$ vs. $38 \mathrm{~min}, p<0.001$ ), more intraoperative blood loss ( $42 \mathrm{~mL}$ vs. $26 \mathrm{~mL}, p=0.008$ ), and longer length of postoperative hospitalization ( 4.5 days vs. 2 days, $p<0.001$ ) than the PTGBD + scheduled LC group. ${ }^{10}$ Postoperative morbidities were considerable higher in the early LC group $(26.7 \%$ vs. $2.7 \%$, $p<0.001)$ with a significantly higher bile leakage rate $(10.7 \%$, $p=0.006) .{ }^{11}$ Despite a higher conversion rate and intraoperative cholangiography requirement, the successful complete cholecystectomy rate in the PTGBD + scheduled LC group was significantly higher than the early LC group $(p<0.001)$. Subtotal cholecystectomies were performed for 13 patients in the early
LC group. ${ }^{11}$ In our opinion, PTGBD is strongly recommended for high-surgical risk patients, especially those who are hemodynamically unstable or those with severe AC (grade II and III), where experienced-laparoscopic surgeons and advanced facilities are not available (Fig. 1). Nevertheless, PTGBD is contraindicated in patients with significant coagulopathy or ascites, or those who are taking anticoagulants/antiplatelets. In addition, this approach has to be performed with special caution in patients with Chilaiditi syndrome, a rare condition in which a part of the transverse colon is sandwiched between the diaphragm and hepatic surface, or with anatomic GB abnormalities.

\section{Endoscopic transpapillary gallbladder drainage}

The current endoscopic transpapillary GB drainage, using a naso-GB tube (5-7 F) or double-pigtail stent that is inserted into the GB, was first described in 1984 by Kozarek. ${ }^{12}$ Transpapillary GB drainage demands special endoscopic skills to introduce a guidewire into the sinuous and thin cystic duct which is usually associated with a stenosis or obstruction by a impacted stone, mass, or occlusion of the cystic duct by a metallic stent. ${ }^{3,13}$ ETGBD accesses the GB lumen through the

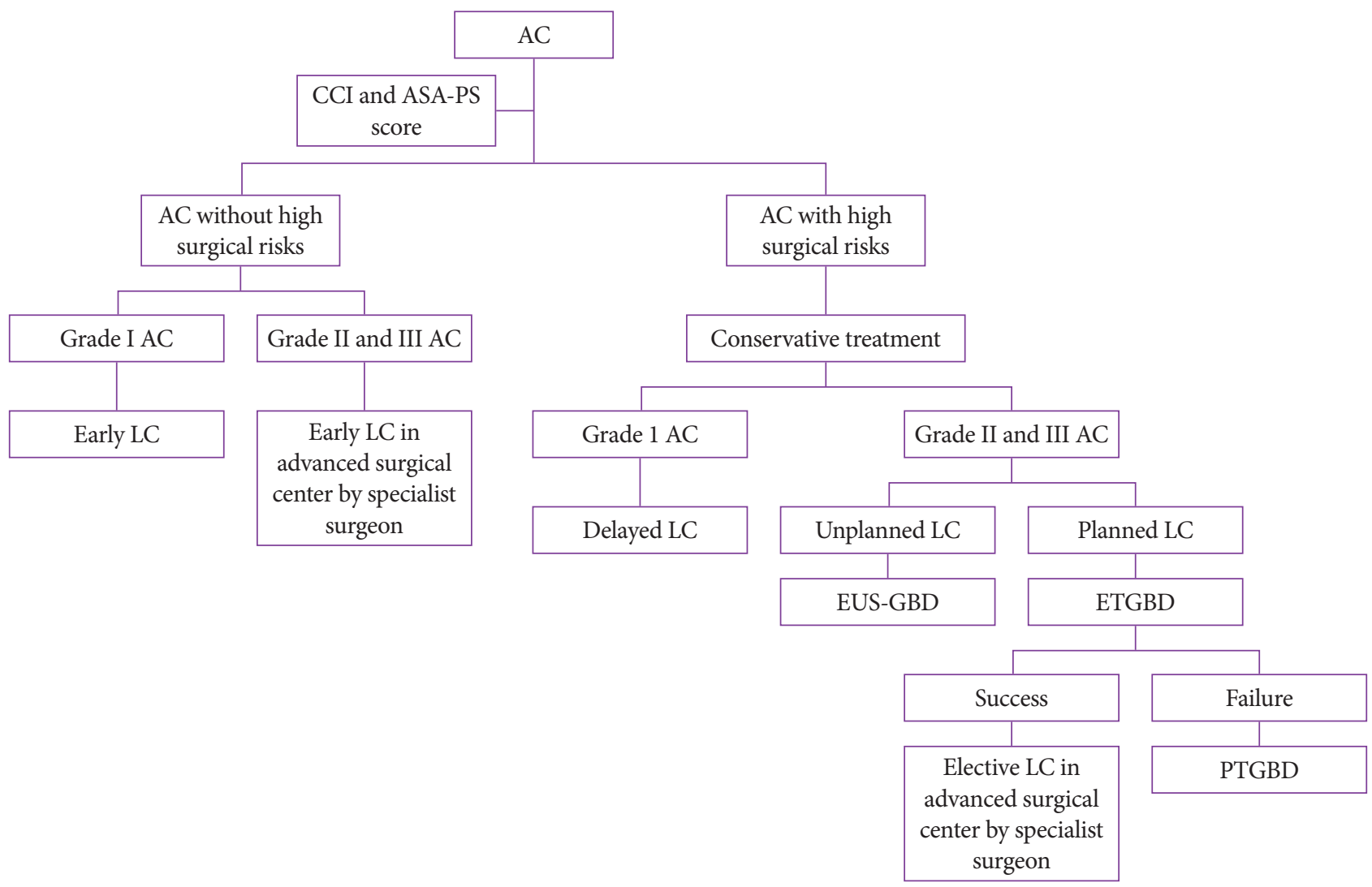

Fig. 1. The algorithm for acute cholecystitis (AC) management is dependent on severity grading. ASA-PS, anesthesiologists physical status classification score; CCI, Charlson comorbidity index; ETGBD, endoscopic transpapillary gallbladder drainage; EUS-GBD, endoscopic ultrasound-guided gallbladder drainage; LC, laparoscopic cholecystectomy; PTGBD, percutaneous transhepatic gallbladder drainage. 
cystic duct by a selective guidewire and placement of a transpapillary and transcystic duct with a double-pigtail (7-10 F) plastic stent, which can be placed indefinitely or taken out by the endoscopist one day prior or at the time of cholecystectomy (Fig. 2A, B). The procedure may not be successful if the cystic duct cannot be demonstrated by fluoroscopic guidance or the guidewire cannot pass the cystic duct into the GB due to its zigzag or very sharp angulation. ${ }^{14}$ The technical success rate of this procedure varies from $50 \%$ to $100 \% .^{13-22}$ It is a good alternative in AC patients who are unsuitable for PTGBD due to contraindications. ETGBD provides at least 6-24 months of stent patency. ${ }^{15} \mathrm{~A}$ multicenter prospective study ${ }^{15}$ included 29 patients who were contraindicated for surgery, all of them underwent ETGBD using a 7 Fr double-pigtail stent to relieve the cystic duct obstruction. During the patient follow-up time (median of 586 days), late complications developed in only $20 \%$ of patients $(n=4)$, including stent migration $(n=2)$ and occlusion $(n=2)$. The overall median of stent patency was 760 days. ${ }^{15}$

Recently, peroral cholangiopancreatoscopy (POCPS) was

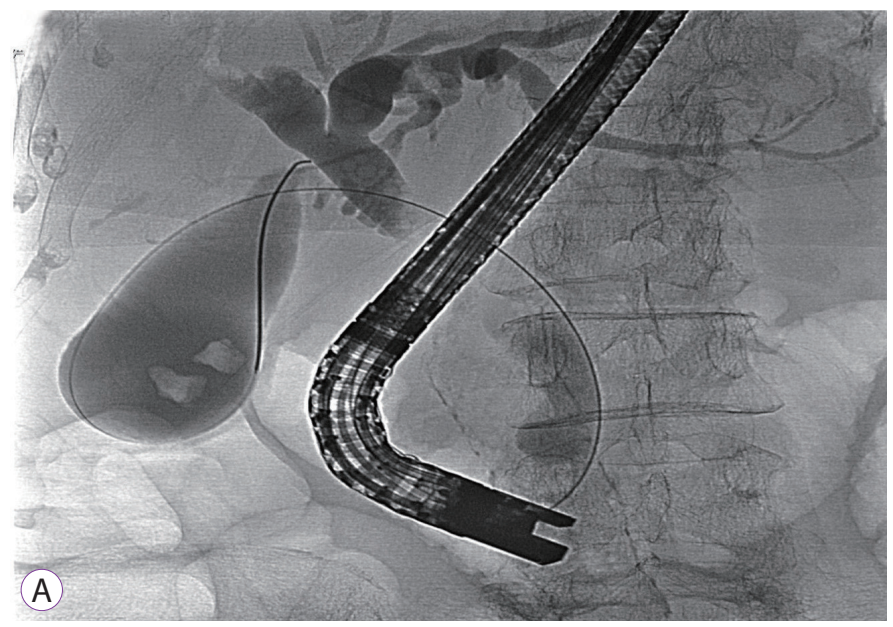

used as a powerful modality to resolve many difficult scenarios in pancreatico-biliary diseases. POCPS-guided cystic duct cannulation can be used as a back-up after failed conventional cystic duct cannulation via fluoroscopic guidance. Endoscopists can directly see the cystic duct orifice by POCPS and a $0.025-0.035$-inch stiff guidewire can be cannulated into the cystic duct from the working channel of the peroral cholangiopancreatoscope. The guidewire is then inserted into the GB under fluoroscopic cholangiogram. In the last step, a $7 \mathrm{Fr}$, $10-15-\mathrm{cm}$ double-pigtail stent is inserted into the GB. ${ }^{16-18}$ The POCPS technique can provide an additional $20 \%$ technical success rate after failed ETGBD under fluoroscopic guidance (Fig. 3A-D). ${ }^{19}$ When POCPS is not available, additional techniques to achieve cystic duct cannulation under fluoroscopic guidance can be performed before considering alternative drainages. In our experience, a bendable tip catheter with a slim and stiff guidewire is helpful to cannulate and traverse a tortuous cystic duct. Another technique using a balloon occluded above the cystic duct take-off, which involves a guidewire passed below the cystic duct and bouncing off the

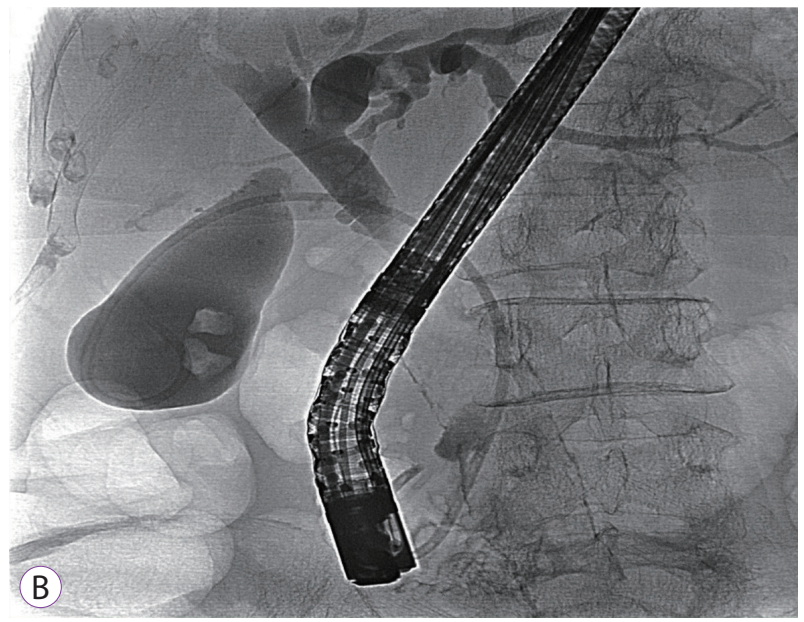

Fig. 2. Endoscopic transpapillary gallbladder drainage (ETGBD) under fluoroscopic guidance. (A) ETGBD accesses the gallbladder lumen through the cystic duct via a selective guidewire. (B) ETGBD placement of a transpapillary, transcystic double-pigtail $7 \mathrm{~F}-15$-cm stent in the duodenum.
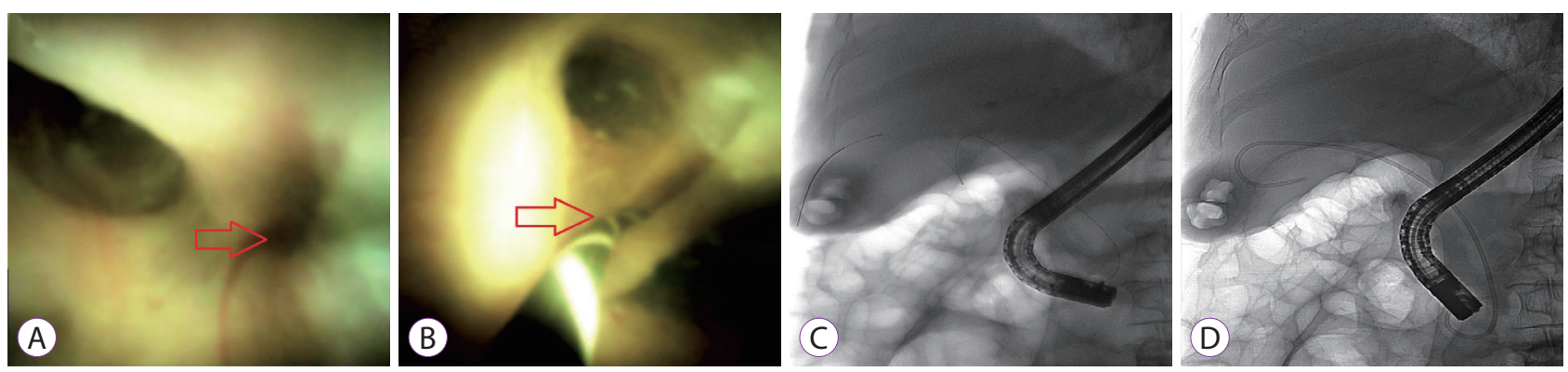

Fig. 3. Endoscopic transpapillary gallbladder drainage with the SpyGlass cholangiopancreatoscopy system. (A) Identifying a slit-like opening (arrow) of the cystic duct using a cholangioscope. (B) Cannulation into the slit-like opening (arrow) by a 0.025 guidewire. (C) Insertion of a zigzag-shaped guidewire in the cystic duct. (D) Placement of a transpapillary, transcystic double-pigtail $7 \mathrm{~F}-15-\mathrm{cm}$ stent into the gallbladder. 
balloon, can sometimes be successful for the cannulation of the cystic duct.

A retrospective review with a total of 172 high-risk surgical patients with AC who were divided into two groups, EUS-GBD $(n=76)$ and ETGBD ( $n=96)$, compared the procedure-related and long-term outcomes between EUS-GBD and ETGBD. In this study, the rate of common bile duct (CBD) stones in AC patients who underwent ETGBD at the time of intervention was higher than those who received the EUS-GBD approach $(29.2 \%$ vs. $7.2 \%, p<0.01)$. ETGBD can be performed in the same ERCP session for imaging modalities and/or laboratory-realized potential CBD stones. This clinical practice reduces the workload burden and results in a more cost-effective treatment for patients. Theoretically, ETGBD can preserve the normal GB structure without the need to create a fistula or adhesion on the GB wall. Moreover, this technique is a suitable indication for patients with serious coagulopathy and thrombocytopenia, notably when endoscopic sphincterotomy is not performed. ${ }^{20}$ Itoi et al. reported a retrospective study of 43 moderate and severe AC patients in which PTGBD was contraindicated or anatomically not feasible. ${ }^{21}$ Without POCPS, ETGBD was successful in $36 \mathrm{pa}-$ tients (84\%) without any serious adverse events and a clinical success rate was observed in 35 patients $(97 \%)^{21}$

In brief, the ETGBD procedure with a double-pigtail stent can be on par with PTGBD in terms of the success rate in both technical issues and clinical outcomes with the additional advantage of preserving the GB texture and its nearby structures. This advantage is helpful for future cholecystectomies in those who are operative candidates. ETGBD is especially suitable for $\mathrm{AC}$ patients who have concomitant $\mathrm{CBD}$ stones. However, this technique demands skillful endoscopists and sometimes needs additional help from POCPS to result in a high technical success rate.

\section{Endoscopic ultrasound-guided gallbladder drainage}

Recently, transmural GB drainage by endoscopic ultrasound-guided access has generated a great interest in the feasibility and effectiveness of comparable approaches that reduce the requirement of PTGBD and ETGBD. EUS-GBD uses a conventional linear array echoendoscope with or without fluoroscopic guidance. Patients are sedated with conscious sedation by intravenous propofol or midazolam and meperidine. EUS-GBD can be performed as a one- or two-step access with the last outcome in which a tract is created between the GB and stomach or duodenum, and subsequently a double-pigtail plastic stent or metallic stent is deployed transluminally. Firstly, a 19-gauge needle is used to puncture the prepyloric area of the stomach or the bulb of the duodenum, to enter the GB whilst any blood vessels and the cystic duct are excluded in the needle path. Bile fluid is then aspirated to confirm puncture of the correct organ and may be used for bacterial cultures. Following this, contrast agents are injected into the GB under fluoroscopy to determine the anatomy of the GB. A $0.025-0.035$-inch guidewire is passed into the GB via the needle and coiled inside the GB. In the one-step access, a hot system which includes an electrocautery-tipped $10 \mathrm{~F}$ catheter containing a lumen-apposing metallic stent (LAMS) with 10$16 \mathrm{~mm}$ effective diameter is used to subsequently cauterize and deploy the LAMS (some experts perform a direct puncture with a hot LAMS system without pre-puncture by a needle. Therefore, this is considered a real one step). In the multistep access, a $6 \mathrm{~F}$ dilator is used after exchanging the needle to dilate the tract. If resistance is encountered while advancing the $6 \mathrm{~F}$ bougie, a $6 \mathrm{~F}$ cystotome is used to create a tract by electrocautery with the pure-cutting mode over the coiled guidewire inside the GB. If there is resistance during advancement of the stent, a 4-6-mm biliary balloon dilator is used to increase the diameter of the tract for the advancement of the stent. It is well advised that the distance between the GB wall and the duodenal or gastric wall is measured and determined to be less than 10-20 $\mathrm{mm}$ to fit between the two anchoring flanges of the LAMS.

Early applications of EUS-GBD used double-pigtail plastic stents or fully covered self-expandable metal biliary stents (FCSEMS), but LAMS has been popularly applied in recent years (Fig. 4A, B). A systematic review from twenty-one studies, ${ }^{23}$ which EUS-GBD was performed by placing either plastic stents or FCSEMS/LAMS. The technical and clinical success rates ranged from $90 \%-100 \%$ in all three stents and the over-
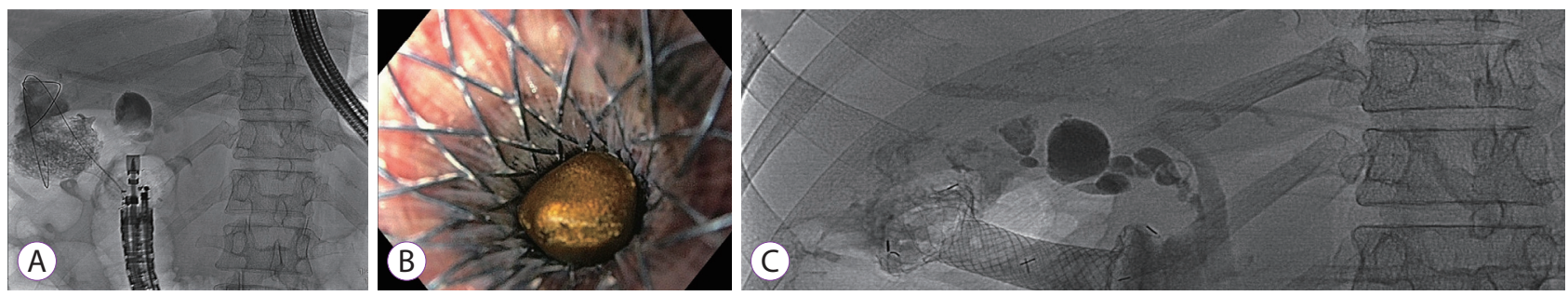

Fig. 4. Endoscopic ultrasound-guided gallbladder drainage (EUS-GBD). (A) EUS-GBD with lumen-apposing metallic stent (LAMS). (B) Gallstones moved into the duodenum via LAMS. (C) LAMS fully expanded with the diameter allowing access to the gallbladder by a slim endoscope. 
all technical and clinical success rates were $95.8 \%$ and $93.4 \%$, respectively. The overall rate of adverse events was $12.0 \%$. However, the rate of adverse events was higher with plastic stents (18\%), whereas it was less than $12 \%$ when FCSEMS and LAMS were used. In detail, bile leakage (4.5\%) typically developed in the plastic stent group due to easy detachment of the GB wall from the gastric or duodenal wall, whereas stent migration $(4.1 \%)$ and occlusion (4.1\%) developed in those who had FCSEMS. ${ }^{23}$

LAMS is an FCSEMS with large flanges at the bilateral ends specifically designed for EUS-guided drainage, which is used for transmural drainage of the pseudocyst and non-adherent fluid collection. The design of this stent supports a strong two-lumen anchorage which can avoid the limitations of FCSEMS. The theoretical advantage of LAMS over other stents is the capacity to fix the GB wall to the gut wall avoiding potential bile leakage once deployed. In addition, the large available diameter of LAMS (10, 15, and $20 \mathrm{~mm}$ ) may allow the insertion of a slim endoscope into the GB for the purpose of future stone removal or lithotripsy (Fig. 4C). EUSGBD with LAMS reportedly has the lowest adverse event rate, probably due to the aforementioned advantages. ${ }^{23}$ Unlike pseudocysts, the GB cavity can be smaller after drainage by LAMS but the GB never collapses. In order to prevent future injury to the inner GB wall when it shrinks, we advocate placing a short double-pigtail stent inside the LAMS for those who plan to have permanent LAMS placement.

Following the Tokyo Guidelines 2018, EUS-GBD has been added as an option for GB drainage in high-risk surgical patients. ${ }^{3}$ Of note, it should be performed in high volume tertiary care centers by skillful endoscopists. ${ }^{3}$ The outcomes of a retrospective, international, multicenter registry on EUS-GBD in AC patients at high risk for cholecystectomy from 13 institutions around the globe demonstrated that unplanned procedural events, such as dislodged guidewires or misdeployment of stents, were significantly more common by endoscopists with experience of fewer than 25 procedures $(p=0.033) .^{24}$

Many studies have compared the technical and clinical outcomes between EUS-GBD and PTGBD. ${ }^{9,25}$ Tyberg et al. conducted a retrospective study including 155 patients from 17 referral centers who received the EUS-GBD $(n=42)$ or PTGBD approach $(n=113)$ for high-risk surgical AC. ${ }^{9}$ Although, the technical success rate was slightly higher in the PTGBD group ( $99 \%$ vs. $95 \%$ ), the clinical success rate was significantly higher in the EUS-GBD group (95\%) than the PTGBD group (86\%). ${ }^{9}$ We speculate that the larger diameter of the stent provided by EUS-GBD, facilitated better drainage. Additionally, the number of patients requiring repeat interventions in the PTGBD group $(n=28,25 \%)$ was significantly higher when compared with the EUS-GBD group $(n=4,10 \%$; $p=0.037)$. The majority of repeat procedures were either from catheter blockage or dislodgement. ${ }^{9}$ Interestingly, EUS-GBD had a potential cost-effectiveness and morbidity benefit by demonstrating a decreased number of re-admissions for interventions. The only downside for EUS-GBD is its lower rates of complete cholecystectomy when compared with PTGBD (5\% vs. $27 \%$, $p=0.003){ }^{9}$ Of note, the study claimed that this was due to differences in the AC etiology between the two groups and not because of EUS-GBD-related GB wall adhesion. Many experts have claimed that LC after EUS-GBD with LAMS was still possible with some difficulties. ${ }^{9}$ Teoh et al. specifically studied the adverse events rate between EUS-GBD and PTGBD. ${ }^{25}$ Among 118 patients with AC who were not candidates for surgery, although, the technical and clinical success rates were

Table 1. The Crucial Outcomes and Suitable Indications of Gallbladder Drainage Procedures

\begin{tabular}{lccc}
\hline & PTGBD & ETGBD & EUS-GBD \\
\hline Technical success rate (\%) & $98-99^{8,9}$ & $50-100^{\mathrm{b}), 13-22}$ & $91.5-100^{\mathrm{c}, 23-25}$ \\
Clinical success rate (\%) & $86-97^{8,9}$ & $76.3-97^{\mathrm{b}), 18,22}$ & $90.1-100^{\mathrm{c}, 23-25}$ \\
Adverse events (\%) & $20-24^{\mathrm{a}, 8,9}$ & $8.7-10^{\mathrm{b}), 15,22}$ & $9.9-18.2^{\mathrm{c}, 23}$ \\
Preserve surrounding structure & Yes & Yes & No \\
Difficulty for delayed LC & No & No & Yes \\
Permanent drainage & No & No & Yes \\
Possible treatment in concomitant CBD stone & No & Yes & No \\
Contraindication in ascites and coagulopathy & Yes & No & Yes \\
\hline
\end{tabular}

CBD, common bile duct; ETGBD, endoscopic transpapillary gallbladder drainage; EUS-GBD, endoscopic ultrasound-guided gallbladder drainage; LC, laparoscopic cholecystectomy; PTGBD, percutaneous transhepatic gallbladder drainage.

${ }^{a)}$ Pain is not involved in this adverse events rate.

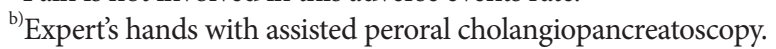

${ }^{c}$ Depend on the type of stent used (plastic stent, fully covered self-expandable metal biliary stent, lumen-apposing metallic stent) and expert's hands. 
similar, 59 AC patients who underwent EUS-GBD had significantly lower overall (32.2\%) and serious (23.7\%) adverse events rate than in the 59 patients who underwent PTGBD (74.6\% and $74.6 \%$, respectively; $p<0.001) .{ }^{25}$ In addition, a total of 16 patients had more than one unplanned admission for problems related to the PTGBD tube which required reintervention or treatment. ${ }^{25} \mathrm{~A}$ systematic review and meta-analysis, including five comparative studies (206 AC patients in the EUS-GBD group vs. $289 \mathrm{AC}$ patients in the PTGBD group), confirmed no statistically significant differences in technical and clinical success rates between the two groups. It is difficult to explain this observation, and we speculate that the different levels of expertise was the causative factor because EUS-GBD was performed mainly by EUS experts whereas PTGBD was performed in a community setting. Moreover, EUS-GBD had fewer adverse events than PTGBD (OR, 0.43) which resulted in fewer reinterventions (OR, $0.16 ; p<0.001 ; I^{2}=32 \%$ ). Of note, this review included a study that defined pain as an adverse event and this could falsely lead to the higher overall adverse events in the PTGBD group. ${ }^{25}$

In our opinion, for patients with $\mathrm{AC}$ who cannot undergo surgery due to malignancy related cholecystitis or poor general condition without a chance for improvement, EUS-GBD is a better alternative than PTGBD since it can reduce the rates of unplanned remission and re-intervention.

A recently published retrospective study analyzed the data obtained from $78 \mathrm{AC}$ patients who were deemed high surgical risk for comparisons with ETGBD. GB drainage was performed by either EUS-GBD $(n=40)$ or ETGBD $(n=38)$. The technical success rate was significantly higher in the EUSGBD group than in the ETGBD group ( $97.5 \%$ vs. $84 \%$, respectively). ${ }^{26}$ The clinical success rate was also significantly higher in the EUS-GBD group (95.0\% vs. $76.3 \%$ ). The recurrent AC rate was also significantly lower in the EUS-GBD group (2.6\%) than in the ETGBD group (18.8\%) on univariate analysis but not in a multiple regression model. ${ }^{26}$

We can conclude that in terms of technical success, clinical success, and the adverse events rate, EUS-GBD is the best choice when compared with PTGBD and ETGBD. This procedure is especially suitable for $\mathrm{AC}$ patients who are indicated for permanent GB drainage. However, we do not recommend this technique for those with scheduled LC. Experienced endosonographers with well-equipped drainage devices are imperative for EUS-GBD success.

\section{CONCLUSIONS}

Based on the literature and available evidence, ETGBD is a safe and effective technique, which preserves the structures around the GB, for GB drainage in moderate and severe AC where surgical expertise is unavailable. In addition, ETGBD should be the first choice for GB drainage when there are concomitant $\mathrm{CBD}$ stones requiring ERCP because the two procedures can be performed in the same ERCP session. A novel POCPS method has improved the cystic duct cannulation success rate in ETGBD, but the failure of cystic duct stent insertion is still possible. Although EUS-GBD has the highest technical and clinical success rates over ETGBD and PTGBD for GB drainage especially when it is performed by experienced therapeutic endoscopists, it may not be the first option if LC is planned. We recommended EUS-GBD to be used as a salvage treatment when ETGBD fails or as the primary treatment in non-surgical candidates. Nevertheless, PTGBD is still the standard approach that maintains its popularity in many institutions particularly when there is no contraindication. PTGBD should be considered as a backup treatment when ETGBD fails and EUS-GBD is not available. The main disadvantage of PTGBD is its high rate of reintervention, therefore it should only be used for temporary GB drainage before the scheduled LC. From the results of many systematic reviews and studies, we have summarized the crucial outcomes and suitable indications of GB drainage procedures (Table 1).

\section{Conflicts of Interest}

The authors have no financial conflicts of interest.

\section{Acknowledgements}

This Research is funded by Chulalongkorn University CU-GRS62-02-30-01 and was supported by the Center of Excellence for Innovation and Endoscopy in Gastrointestinal Oncology, Faculty of Medicine, Chulalongkorn University.

ORCID

Khanh Cong Pham: https://orcid.org/0000-0001-9945-1079

\section{REFERENCES}

1. Yokoe M, Takada T, Hwang TL, et al. Descriptive review of acute cholecystitis: Japan-Taiwan collaborative epidemiological study. J Hepatobiliary Pancreat Sci 2017;24:319-328.

2. Ansaloni L, Pisano M, Coccolini F, et al. 2016 WSES guidelines on acute calculous cholecystitis. World J Emerg Surg 2016;11:25.

3. Mori Y, Itoi T, Baron TH, et al. Tokyo guidelines 2018: management strategies for gallbladder drainage in patients with acute cholecystitis (with videos). J Hepatobiliary Pancreat Sci 2018;25:87-95.

4. Cao AM, Eslick GD, Cox MR. Early laparoscopic cholecystectomy is superior to delayed acute cholecystitis: a meta-analysis of case-control studies. Surg Endosc 2016;30:1172-1182.

5. Tsuyuguchi T, Itoi T, Takada T, et al. TG13 indications and techniques for gallbladder drainage in acute cholecystitis (with videos). J Hepatobiliary Pancreat Sci 2013;20:81-88.

6. Okamoto K, Suzuki K, Takada T, et al. Tokyo guidelines 2018: flowchart for the management of acute cholecystitis. J Hepatobiliary Pancreat Sci 
2018;25:55-72.

7. Yokoe M, Hata J, Takada T, et al. Tokyo guidelines 2018: diagnostic criteria and severity grading of acute cholecystitis (with videos). J Hepatobiliary Pancreat Sci 2018;25:41-54.

8. Siddiqui A, Kunda R, Tyberg A, et al. Three-way comparative study of endoscopic ultrasound-guided transmural gallbladder drainage using lumen-apposing metal stents versus endoscopic transpapillary drainage versus percutaneous cholecystostomy for gallbladder drainage in highrisk surgical patients with acute cholecystitis: clinical outcomes and success in an international, multicenter study. Surg Endosc 2019;33:12601270 .

9. Tyberg A, Saumoy M, Sequeiros EV, et al. EUS-guided versus percutaneous gallbladder drainage: isn't it time to convert? J Clin Gastroenterol 2018;52:79-84.

10. Lee R, Ha H, Han YS, Kwon HJ, Ryeom H, Chun JM. Percutaneous transhepatic gallbladder drainage followed by elective laparoscopic cholecystectomy for patients with moderate to severe acute cholecystitis. Medicine (Baltimore) 2017;96:e8533.

11. Tan HY, Jiang DD, Li J, He K, Yang K. Percutaneous transhepatic gallbladder drainage combined with laparoscopic cholecystectomy: a meta-analysis of randomized controlled trials. J Laparoendosc Adv Surg Tech A 2018;28:248-255.

12. Kozarek RA. Selective cannulation of the cystic duct at time of ERCP. J Clin Gastroenterol 1984;6:37-40.

13. Pannala R, Petersen BT, Gostout CJ, Topazian MD, Levy MJ, Baron TH. Endoscopic transpapillary gallbladder drainage: 10-year single center experience. Minerva Gastroenterol Dietol 2008;54:107-113.

14. Barkay O, Bucksot L, Sherman S. Endoscopic transpapillary gallbladder drainage with the SpyGlass cholangiopancreatoscopy system. Gastrointest Endosc 2009;70:1039-1040.

15. Lee TH, Park DH, Lee SS, et al. Outcomes of endoscopic transpapillary gallbladder stenting for symptomatic gallbladder diseases: a multicenter prospective follow-up study. Endoscopy 2011;43:702-708.

16. Shin JU, Lee JK, Kim KM, Lee KH, Lee KT. Endoscopic naso-gallbladder drainage by using cholangioscopy for acute cholecystitis combined with cholangitis or choledocholithiasis (with video). Gastrointest Endosc 2012;76:1052-1055.
17. Kurihara T, Yasuda I, Isayama H, et al. Diagnostic and therapeutic single-operator cholangiopancreatoscopy in biliopancreatic diseases: prospective multicenter study in Japan. World J Gastroenterol 2016;22:18911901.

18. Ramchandani M, Reddy DN, Lakhtakia S, et al. Per oral cholangiopancreatoscopy in pancreatico biliary diseases--expert consensus statements. World J Gastroenterol 2015;21:4722-4734.

19. Teeratorn N, Ridtitid W, Piyachaturawat P, Kongkam P, Rerknimitr R. Efficacy of digital cholangioscopy-assisted cystic duct cannulation for transpapillary gallbladder stenting. Gastrointest Endosc 2019;89(6 Suppl):AB254-AB255.

20. Oh D, Song TJ, Cho DH, et al. EUS-guided cholecystostomy versus endoscopic transpapillary cholecystostomy for acute cholecystitis in highrisk surgical patients. Gastrointest Endosc 2019;89:289-298.

21. Itoi T, Sofuni A, Itokawa F, et al. Endoscopic transpapillary gallbladder drainage in patients with acute cholecystitis in whom percutaneous transhepatic approach is contraindicated or anatomically impossible (with video). Gastrointest Endosc 2008;68:455-460.

22. Khan MA, Atiq O, Kubiliun N, et al. Efficacy and safety of endoscopic gallbladder drainage in acute cholecystitis: is it better than percutaneous gallbladder drainage? Gastrointest Endosc 2017;85:76-87.e3.

23. Anderloni A, Buda A, Vieceli F, Khashab MA, Hassan C, Repici A. Endoscopic ultrasound-guided transmural stenting for gallbladder drainage in high-risk patients with acute cholecystitis: a systematic review and pooled analysis. Surg Endosc 2016;30:5200-5208.

24. Teoh AY, Perez-Miranda M, Kunda R, et al. Outcomes of an international multicenter registry on EUS-guided gallbladder drainage in patients at high risk for cholecystectomy. Endosc Int Open 2019;7:E964-E973.

25. Teoh AYB, Serna C, Penas I, et al. Endoscopic ultrasound-guided gallbladder drainage reduces adverse events compared with percutaneous cholecystostomy in patients who are unfit for cholecystectomy. Endoscopy 2017;49:130-138.

26. Higa JT, Sahar N, Kozarek RA, et al. EUS-guided gallbladder drainage with a lumen-apposing metal stent versus endoscopic transpapillary gallbladder drainage for the treatment of acute cholecystitis (with videos). Gastrointest Endosc 2019;90:483-492. 